University of Nebraska - Lincoln

DigitalCommons@University of Nebraska - Lincoln

Faculty Papers and Publications in Animal

Science

Animal Science Department

April 2005

\title{
Physical mapping of genes in the porcine ovarian transcriptome
}

\author{
A. R. Caetano \\ University of Nebraska-Lincoln \\ J. B. Edeal \\ University of Nebraska-Lincoln \\ K. Burns \\ University of Nebraska-Lincoln \\ R. K. Johnson \\ University of Nebraska-Lincoln, rjohnson5@unl.edu \\ C. K. Tuggle \\ lowa State University, IA, \\ See next page for additional authors
}

Follow this and additional works at: https://digitalcommons.unl.edu/animalscifacpub

Part of the Animal Sciences Commons

Caetano, A. R.; Edeal, J. B.; Burns, K.; Johnson, R. K.; Tuggle, C. K.; and Pomp, Daniel, "Physical mapping of genes in the porcine ovarian transcriptome" (2005). Faculty Papers and Publications in Animal Science.

74.

https://digitalcommons.unl.edu/animalscifacpub/74

This Article is brought to you for free and open access by the Animal Science Department at DigitalCommons@University of Nebraska - Lincoln. It has been accepted for inclusion in Faculty Papers and Publications in Animal Science by an authorized administrator of DigitalCommons@University of Nebraska - Lincoln. 


\section{Authors}

A. R. Caetano, J. B. Edeal, K. Burns, R. K. Johnson, C. K. Tuggle, and Daniel Pomp 
Published in Animal Genetics 36:4 (2005), 322-330; doi:10.1111/j.1365-2052.2005.01306.x Copyright (C) 2005 International Society for Animal Genetics; published by Blackwell Publishing. Used by permission. http://www.blackwell-synergy.com/loi/AGE

Accepted for publication April 1, 2005

\title{
Physical mapping of genes in the porcine ovarian transcriptome
}

\author{
A. R. Caetano*§, J. B. Edeal ${ }^{*}$, K. Burns*, R. K. Johnson*, C. K. Tuggle ${ }^{\dagger}$, and D. Pomp*‡ \\ * Department of Animal Science, University of Nebraska-Lincoln, Lincoln, NE, USA. \\ † Department of Animal Science, lowa State University, Ames, IA, USA \\ § Present address: A. R. Caetano, Embrapa Recursos Genéticos e Biotecnologia, Parque Estação Biológica, Final \\ Av. W/5 Norte, Brasília-DF, CP 02372, 70770-900, Brasil. \\ ‡Corresponding author; Email: dpomp@unl.edu
}

\begin{abstract}
Reproductive efficiency and associated traits are of major economic importance to the swine industry and have been more difficult to improve genetically than other production traits. Integration of phenotypical data with gene mapping and expression studies provides a powerful approach for dissection of the genetic basis regulating complex traits. We developed a total of 101 polymerase chain reaction-based markers, representing 91 unique genes, for expressed sequence tags previously reported to be putatively differentially expressed in the porcine ovarian transcriptome of a swine line selected on an index of high ovulation rate and embryonic survival. These were subsequently used in physical mapping experiments with a porcine radiation hybrid and somatic cell hybrid panels. Our results increased the information content of the porcine physical map useful for comparative mapping by c. $10 \%$. Moreover, the mapped genes are likely to be biologically relevant to the molecular mechanisms that control ovulation rate in the pig. A total of 12 differentially expressed genes were mapped to regions previously reported to contain quantitative trait loci affecting swine ovulation rate.
\end{abstract}

Keywords: expressed sequence tags, expression profiling, mapping, ovarian follicle, quantitative trait loci, radiation hybrid, swine.

\section{Introduction}

Reproduction is of central importance to the pork industry, and significant resources have been devoted to understand biological phenomena that could lead to further genetic improvement of reproductive efficiency in commercial swine lines. Although map-based approaches have been shown to be powerful at identifying genes that affect production traits in livestock (e.g. Fujii et al. 1991; McPherron and Lee 1997; Milan et al. 2000; Page et al. 2002; Grisart et al. 2004), quantitative trait loci (QTL) mapping methodology has not been very fruitful in the studies of reproductive traits, providing inconsistent results with low resolution, that have thus far not allowed for identification of the underlying genes.

Integrated approaches, merging mapping information with gene-expression data, have been proposed as a viable alternative to dissect the molecular basis of complex traits (Wayne and McIntyre 2002; Schadt et al. 2003; Pomp et al. 2004).
Such approaches require high-density maps, saturated with known genes and expressed sequence tags (ESTs), as well as reagents for high throughput gene expression analysis. In the pig, several studies have generated and/or used genomic tools to study the molecular basis of economically important traits (e.g. Rohrer et al. 1996; Fahrenkrug et al. 2002; Rink et al. 2002; Caetano et al. 2003, 2004; Tuggle et al. 2003, reviewed in Rothschild 2003). Although specific large-scale resources have been created to study swine reproductive traits (see Pomp et al. 2001; Tuggle et al. 2003; Rohrer 2004), these still require further development. In support of this, the information content of the swine radiation hybrid (RH) map has been significantly increased by the recent addition of a large number of ESTs (Rink et al. 2002; Tuggle et al. 2003), as well as large-scale comparative flanking sequence annotation of microsatellite loci (Robic et al. 2003).

Caetano et al. (2004) conducted an expression-profiling study to identify genes that are differentially expressed in ova- 
ries and ovarian follicles of sows from swine lines selected for an index of high ovulation rate and embryo survival (Johnson et al. 1999), during the follicular phase of the estrous cycle. We now report on the physical mapping of 91 of the genes identified as differentially expressed in that study.

\section{Materials and methods}

\section{Source of EST sequences}

Caetano et al. (2003) generated 3479 unique ESTs by sequencing clones from a normalized ovarian follicle cDNA library. These clones, in addition to a few other ovarian follicle-derived ESTs, were used to build a 9216 feature cDNA microarray that was subsequently used in expression profiling experiments to compare mRNA levels from ovarian follicles and from ovaries between a swine line selected for enhanced reproduction and its randomly selected control line (Caetano et al. 2004). Using very strict statistical analyses, 131 probes representing 106 unique genes were found to be significantly differentially expressed (Caetano et al. 2004). Sequences of ESTs for these genes (Caetano et al. 2003) were used to design primers for RH and somatic cell hybrid (SCH) mapping.

\section{PCR primer design and optimization of amplification}

Design of polymerase chain reaction (PCR) primers was as previously described (Tuggle et al. 2003). Briefly, primers were designed with Primer3 (http://0-www-genome.wi.mit. edu.library.unl.edu:80/cgi-bin/primer/primer3_www.cgi) with some changes in the default parameters (primer size of $25 \mathrm{bp}$, melting temperature of $65^{\circ} \mathrm{C}$, and maximum difference between $T_{\mathrm{m}}$ of the left and right primers of $5{ }^{\circ} \mathrm{C}$ ). Each primer set (Table 1) was tested against mouse, hamster and pig DNA in $10-\mu 1$ reactions containing $25 \mathrm{ng}$ of genomic DNA, $1.5 \mathrm{~mm}$ $\mathrm{MgCl}_{2}, 50 \mu \mathrm{m}$ of each dNTP, $2 \mu \mathrm{m}$ each primer, $1 \mathrm{U}$ Taq polymerase, $1 \mathrm{X}$ reaction buffer (Promega, Madison, WI, USA), and 1X Rediload (Invitrogen, Carlsbad, CA, USA). Cycling conditions used with each primer set are described in Table 2. Amplification products were visualized after electrophoresis in ethidium bromide-stained 4\% high:low (3:1) melting agarose gels. Primers that did not amplify a pig-specific fragment were redesigned and retested at least once.

\section{$\mathrm{SCH}$ panel and $\mathrm{RH}$ panel analysis}

Primers that generated pig-specific PCR products were used to type the INRA-University of Minnesota porcine radiation hybrid panel (IMpRH) panel (Yerle et al. 1998) using optimized conditions (Table 2). Data were initially evaluated using the IMpRH database (http://0-imprh.toulouse. inra.fr.library.unl.edu:80/) to determine map positions. Those ESTs with LOD scores $\geq 6.0$ were submitted to the IMpRH database and those with scores $<6.0$ were rescored and results were resubmitted. Primers for all ESTs with final LOD scores $<6.0$ were subsequently used to type the INRA swine SCH panel (SCHP) (Yerle et al. 1996) with the same optimized PCR conditions. Data were submitted online (http://0www.toulouse.inra.fr.library.unl.edu/lgc/pig/hybrid.htm) and regional assignments were obtained using the INRA database (http://0-www.toulouse.inra.fr.library.unl.edu/lgc/pig/per/pcr. $\underline{\mathrm{htm}})$.

\section{Results}

As expected, the mapped genes are distributed across all pig chromosomes. Results for six of the ESTs mapped with the RH and SCHPs were discordant. Estimated positions of human orthologues, based on the UCSC Genome Browser (http://genome.ucsc.edu/; assembly of July 2003), are listed in Table 2, when a link was available in the TIGR Pig Gene Index [SsGI (v8.0); Quackenbush et al. 2000] for the tentative consensus sequence containing the corresponding EST. The expected position of human orthologues was not available for 17 of the mapped ESTs. Predicted positions of human orthologues, based on the human-pig chromosomal painting data (Goureau et al. 1996) and results of single locus physical mapping (http://0-www.toulouse.inra.fr.library.unl.edu/ lgc/pig/cyto/cyto.htm), are provided in Table 2. The expected cytogenetic positions of the human orthologues were in agreement with $85 \%$ of the mapped ESTs, based on comparisons of both of these databases. The human physical position of three additional ESTs was in agreement when we considered the expected human cytogenetic position based on the pig SCHP results.

\section{Discussion}

Development of RH mapping panels has proven to be an extremely useful tool for rapidly constructing high-density physical maps of mammalian genomes. This method is more amenable to high throughput mapping relative to efforts based on linkage, especially for species with limited SNP resources. The latest version of the published porcine RH map was based on 1058 EST-derived markers (Rink et al. 2002). The current effort resulted in addition of 101 ESTs, representing 91 unique genes, to the porcine RH map, improving its density and coverage, and increasing the information content useful for comparative mapping. Moreover, the ESTs mapped in this study represent genes found to be putatively differentially expressed in ovaries and ovarian follicles of pigs selected for enhanced female reproduction (Caetano et al. 2004), and are thus likely to play important roles in the biological processes that control ovulation rate in swine.

The systematic approach adopted to design primers and optimize PCR conditions was successful. Less than a quarter of the primer sets designed failed to produce amplicons useful 
Table 1 Primer pairs for mapping swine expressed sequence tags.

\begin{tabular}{|c|c|c|c|}
\hline $\begin{array}{l}\text { Genbank } \\
\text { accession } \\
\text { no. }\end{array}$ & 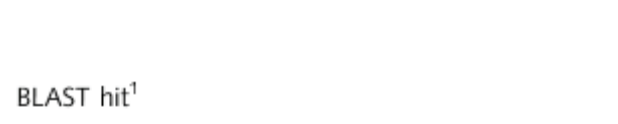 & Forward primer $\left(5^{\prime} \rightarrow 3^{\prime}\right)$ & Reverse primer $\left(5^{\prime} \rightarrow 3^{\prime}\right)$ \\
\hline AW507066 & Cytochrome P450 side chain cleavage (CP450SSC) & GATGTGGCCCTTCTCTTTAAGTTCG & TGGAACATCTGGTAGACAGCATCAA \\
\hline AW507072 & Cytochrome c oxidase subunit 3 & ATAGTAAACCCAAGCCCATGACCAC & TGATGGGCTCAGGTAATGGATACTC \\
\hline AW574381 & Cytochrome B & TTTATCCTGCСАTTCATCATTACCG & ATTATGCCTCGTTGTTTGGATGTGT \\
\hline AW574403 & Cytochrome B and tRNA-Thr and tRNA-Pro & TGGCCCTCCTTTTCTGGTTT & ACCCGTAGAACACCCGTTCA \\
\hline AW584050 & NADH dehydrogenase subunit 4, mRNA sequence & ATGGGGGTAGGGCTAGGTTT & GAAGTTTCATAGGGGCCACA \\
\hline AW584057 & Ferritin & TGGCTTTGAAGAACTTTGCCAAATA & GCTTAGGGCTTAGCTCTCACTGCTC \\
\hline BI180977 & Pro-1(I) collagen chain (Col1A1) & CTTCCCAGAACATCACCTACCACTG & CСAAAGTCCATGTGAAATTGTCTCC \\
\hline BI180991 & Actin-related protein 3 , yeast (ARP3) & ATCCAAGATTTGATGGGATTTATCG & ACAGAACTGCAATACTGGTCCAACA \\
\hline BI181040 & Human chromosome 1q21 BAC $236 \mathrm{c} 22$ & TTTCTTAGGCCATTCCAGTGCATAG & GTCAGGCTAGGGCAGAACGTTTAGT \\
\hline BI181041 & Low density lipoprotein-related protein 1 (LRP1) & AATGGGAAGCGACTGGACAA & GCTCACACTTGTCGССTGTG \\
\hline $\mathrm{BI} 181067$ & Alpha-2-macroglobulin (A2M) i & ACGGCTTCCGGTGTAACTGA & TCCCATTTGCTTTGGAGGTG \\
\hline BI181144 & Pro-1(I) collagen chain (Col1A1) & CAAAGTCTTCTGCAACATGGAGACA & TCGTAGATCACGCTGTAGGTGAAGC \\
\hline BI181194 & $60 S$ ribosomal protein L4 & CTGAAAGTGCTTTCCGCAAGTTAGA & СТTСТСТACСАCGGGCTTСTTGTCT \\
\hline BI181228 & Selenium-binding protein 1 (SELENBP1) & ACTTGGTCGGTAGAGAAGGTGATCC & ATGAGATCGGGGTAAAACTGCTTGT \\
\hline BI181240 & Heat shock protein, DNAJ-like 2 (HSJ2) & GCAGCAAATAAAATGCCAGCAGTAA & CTTGACAACCGAACCATAGTCATCA \\
\hline BI181249 & Sus scrofa microsatellite, locus SO658 & ACCCGAACTCCAACTAGGTCAACTC & GAGGTTTGAACACCAGCAATGTCTT \\
\hline BI181263 & Cytochrome P450 17- $\alpha$-hydroxylase (CYP17) & AAGTACCCCAGGAGTCTCCCAGTC & GTAGTCTTGGAACCCAGACGAAAGG \\
\hline BI181277 & Cathepsin L & TGGAAGGATTCATGGCTTGC & TGCCAACGACACTGGTTACG \\
\hline BI181293 & No match & TTGAAGAAAATGCTTAGCTGCGAAA & GTGAGAAGTGTAAATCACCCCTTGG \\
\hline BI181366 & Cytosolic dihydrodiol dehydrogenase 3 & TTTTTGACTTTGAATTGCCTCCAGA & TGCACCTTCTGTGTCTAATGGGACT \\
\hline BI181384 & Homo sapiens cDNA FLJ13813 & ССАСTGTGGATGTTGACCСTAAGTC & GGTGGATGACCTAAAACAGGAGAGG \\
\hline BI181387 & Tissue inhibitor of metalloproteinase 3 (TIMP3) & GGCCGCTCTGTATTTCATTCATATC & TTAGGAGAAATCATGGCTTCCCAGT \\
\hline BI181421 & 3 - $\beta$-hydroxysteroid dehydrogenase (3BHSD) & GGAGCTGCGTGAAGGGATACATACT & TCTGCTTCTCGCCACCAGAAC \\
\hline BI181431 & Sus scrofa scavenger receptor (SCARB1) & CCAGCCСCTGTAGAGATTTCAGAGA & TGGGGAGAGTCTTGACAACAAAGAG \\
\hline BI181480 & Plasminogen activator inhibitor-1 & AACATTTTTCACTGAAGGGCATGGT & GGGTTGAAGGGAGACAGAGTTGTTT \\
\hline BI181579 & Ubiquitin carboxyl-terminal esterase L1 (UCHL1) & TTGCGAAGATGCAGCTCAAA & ACCGAGCCCAAAGACTCCTC \\
\hline BI181663 & Protective protein for $\beta$-galactosidase (PPGB) & АСTCTGGCCGTGTTGGTCAT & AGAGCCTGTTCCCCAGAAGG \\
\hline BI181686 & Connective tissue growth factor & GCTATTGGGTGTCATTGAGAAAGGA & TGGTGTTCAGAATGGAGGCTAACAT \\
\hline BI181696 & Regulator of G-protein signalling 2, $24 \mathrm{kDa}$ (RGS2) & GAAGCCAGGAGCAGTCTAGGAGAAG & АCTCATCCTGTGACACACAGCAACT \\
\hline BI181761 & Collagen, type I, alpha-1 (COL1A1) & CTGAGCCAGCAGATCGAGAACAT & GGGAAGCCTCAGTGGACATCAG \\
\hline BI181785 & Homo sapiens clone 23785 mRNA & AАCATTCATCTCCAAGAACTGCTTCA & CCAGAGAAACAAAATGGCAAACAGT \\
\hline $\mathrm{BI} 181787$ & Calpain I light subunit & GGGCTAAAGTCCAGGTTAGAGCAGA & GGGAGGACTCTGTTCAGGAAGGAAT \\
\hline BI181791 & Homo sapiens hypothetical protein MGC11256 & GTGTCTGCACACAGGCATCC & GTCCCGAGGGGTGTAAGGAG \\
\hline BI181803 & Cytochrome P450 side chain cleavage & ATTTATTGCCTTCATGGGAGTGGAT & GAGCTCGAGATGACTCTCTTCCTCA \\
\hline BI181842 & Phospholipid transfer protein (PLTP) & GAGCAAGAACTGGAGACCATTACCA & CGAGGTGATGAAAGTGGACAGGAAT \\
\hline $\mathrm{BI} 181891$ & Serpentine receptor (CYT28) & CTGATGACCACGAGGGACTCTGTAG & CCCAGAACGTCTACACTAAGCCTGA \\
\hline BI182004 & Retinoic acid receptor responder 1 & ACTAGTCGGATTCGTTTCTGCTGTG & CCACTGTCAAGAGCTACAGACACCA \\
\hline BI182007 & Putative lysophosphatidic acid acyltransferase & CGAGAGCTCCTGCATACGACAGT & ATTCAAGGAGGAGCAGCGGAAG \\
\hline BI182045 & Tissue inhibitor of metalloproteinase 3 (TIMP3) & GACAGGGTTTTCTCTGGTTGGTTTC & СССTAGGAGACAAGACTCTCCCTGA \\
\hline BI182047 & No match & CTGATCAGAAAATCACCCCACACTC & GCTGCCCTGAAGTTACAGAAGGTG \\
\hline BI182064 & A kinase (PRKA) anchor protein 2 (AKAP2) & AGTGGTGTCTGACTGCAGTTGATTG & СТGCTGCСТСТTCACAAACGTTAAA \\
\hline BI182164 & Human cDNA FLJ11658 fis & GCTCACTCTCTGTCCCAGAACCTTA & TGTGATGGCCTGTGACTCTCCTATT \\
\hline BI182214 & Cathepsin U (CTSU) & AACTGCTGTGAAGAATCAGGGTCAC & GGATTCATGGCTTGCATCTATACCA \\
\hline BI182232 & Heat shock 10 kDa protein 1 (HSPE1) & GAAAGAAGTGCAGCTGAAACGGTAA & CTGGATGTCATCAGTTTGGATATCATT \\
\hline BI182250 & Annexin A2 (ANXA2) & GACACTAAGGCCGTCCTCCA & GTGGTGGGGATGACTGAAGC \\
\hline BI182336 & Cytochrome P450 17- $\alpha$-hydroxylase (CYP17) & AGTCAGGAGGTACTCCCCTCAGTGT & CATTGGCGAATTTACCATTGACAAG \\
\hline BI182401 & No match & TTACCGССТСТСТССССТTC & TGATGTGGGGTCGGGTTTAG \\
\hline BI182422 & Pilin-like transcription factor & TTCAAACCAAGGAAACACTGACCAT & ССТСТTСТСGTTTGАССТСТСАССТ \\
\hline BI182428 & Homo sapiens CDNA FLJ22155 & GTTTCCGCTTCCTGGTCTTGTAAA & TTTCCATCTCTAGAAAATGGCATGG \\
\hline BI182474 & Cysteine dioxygenase, type I (CDO1) & GGGCTCTACTGGTTTCACCCTCTAA & TTTGGTTTGTTGTGGGTTTGGATAC \\
\hline BI182487 & Ribosomal protein S4, X-linked (RPS4X) & TTACACCTGAGGAGGCCAAGTACAA & СТСТTGTCTCTCTCСTCAGCAATGG \\
\hline BI182556 & Thymosin, beta-4, X-chromosome (TMSB4X) & СТTССТССТTСАССАACATGCAA & AACCATGTCTGACAAACCCGATATG \\
\hline BI182567 & NDR1-related protein NDR2 (NDR2) & AAGCTGATAGGGCTTCTCATGAACC & TTACAGCAGACACTGTGGGAGACAG \\
\hline BI182727 & Porcine endogenous retrovirus PERV-MSL & GAGTGTGGAGTCGGGACAGC & TCAAATTGGTTGCGCCATAA \\
\hline
\end{tabular}


Table 1 Continued.

\begin{tabular}{|c|c|c|c|}
\hline $\begin{array}{l}\text { Genbank } \\
\text { accession } \\
\text { no. }\end{array}$ & BLAST hit $^{1}$ & Forward primer $\left(5^{\prime} \rightarrow 3^{\prime}\right)$ & Reverse primer $\left(5^{\prime} \rightarrow 3^{\prime}\right)$ \\
\hline BI182751 & Ras homologue gene family, member B (ARHB) & TCAGGGAACTCGTCCTTACTGAACA & СTAGGCCGGGTCTGCTACTCTAGTC \\
\hline $\mathrm{BI} 182872$ & Calpain I light subunit & TAGGTACACGGGGTCAGTCTAATGC & САATTGGTCTGCTCTAACGTGGACT \\
\hline Bl182881 & Growth factor receptor-bound protein 14 (GRB14) & TGAAGAGGCAGTGGGGCTAC & AATCCCACTGAGGCCCTTTC \\
\hline BI183145 & $\mathrm{H} 2 \mathrm{~A}$ histone family, member $\mathrm{Z}$ (H2AFZ) & TCCACCGAAGTGGAAATAATGTCTG & CATCCACAAATCTCTCATTGGGAAG \\
\hline BI183240 & Sus scrofa Ig gamma $2 a(I G G 2 A)$ & AGACTTCTGGGTGTAGTGGTTGTGC & AAGGAGTTCAAGTGCAAGGTCAACA \\
\hline $\mathrm{BI} 183427$ & CD83 antigen & AGACTGTTGGCAACTACAAGCAAGC & ATAAGGAAACACAACAGCTGCCAAA \\
\hline BI183514 & Tropomyosin 2 (beta) (TPM2) & GTCTGGTGAATCTCCACGTTCTCCT & TGTACTGATGGCATGTGTCTGTTCC \\
\hline BI183574 & Interferon-stimulated protein (ISG15) & GGTGCACATAGGCTTGAGGTCATAC & TAGGGAACTGAAGGTGAAGATGCTG \\
\hline BI183661 & Homo sapiens RGC32 protein (RGC32) & TGCTACGTTTTAAGAGAAAATACCA & AAAAATGCCTTTGGGCTCCT \\
\hline BI183694 & Homo sapiens BORG2 protein (BORG2) & TCATGTTGCCCTTATTGTCACCAGT & TTTATCCATGACATTCAGCACCTCA \\
\hline BI183769 & Apolipoprotein E receptor 2 (ApoER2) & CAAAGCAGTAAACCAAATCCCAGTG & TTAGGACCCTTGGGTTGAAGTCATT \\
\hline BI183910 & BTG family, member 2 (BTG2) & GCACTGACAGAGCACTACAAACACC & ACGGCAGGCAGAATGAGTACAGTAG \\
\hline BI183974 & Tissue inhibitor of metalloproteinase-3 (TIMP3) & TGAAGTTTCCTTGCTGAAAAGTTGG & CTGGCTGCCTGCACAGTAGAAGTAT \\
\hline BI184102 & Heat shock 70 kDa protein 8 (HSPA8) & TGTGGATAAGAGCACAGGAAAGGAG & ССTGCACTCTGGTAGAGCTTGGTAA \\
\hline BI184141 & TNF, alpha-induced protein 1 (TNFAIP1) & GTGGTTAGCAGGCTTCTGGTTTTCT & CTCCCCGTTGAGGTATTTTAATGCT \\
\hline BI184356 & Complement component $1, \mathrm{q}$, alpha (C1QA) & CTTAAAGGAGACCAGGGGGAGTCTG & AAGAGTCCCTTGCTGTTGAAGTCAC \\
\hline BI184390 & Integrin-linked kinase (ILK) & ССТАСТTGTССТGСАТСТTСТССАG & ACTCAACAGTCGCAGTGTCATGATT \\
\hline BI184392 & No match & ССTGGCTCTCCTCAGGGTTT & AACCCCCAGCATCCAAGAAT \\
\hline BI184415 & Cytolytic trigger molecule G7 & TCCATGTGACTTTGCCATTCC & TTCTGCCTGGTGATGGGATT \\
\hline BI184594 & Collagenase inhibitor & TTTCATCATCTTGGAGAAAGACAGGT & ATGGGAGGGACTGTTCTTGAGTCAC \\
\hline BI184598 & Dihydrodiol dehydrogenase-2 (DD2) & ATTCCACCTGGTTGCAGACG & GGCGGCTCTGGAGAAGTGTA \\
\hline BI184629 & Homo sapiens mitogen-inducible gene 6 (MIG-6) & GCTCAAATAACATCACTCAGCAGTGTC & ССTCTTCAGTATTTCCTGGGGATTG \\
\hline BI184678 & Homo sapiens cDNA FLJ22353 & GCTGGAGCTGCTCTTTGATTATGAG & CTCATCGGCCCTTGAGTAGAGGTAG \\
\hline BI184717 & Homo sapies syndecan 4 (SDC4) & ATGGCTGGAGGGAAGGGACAT & ACACTAACCACATTTCAAGGGCTCA \\
\hline BI184862 & $\begin{array}{l}\text { Lysosomal associated protein } \\
\text { transmembrane 4-beta (LAPTM4B) }\end{array}$ & GCTGTGGCTTTTGAACACAGG & СТСТССТGCAAGGCACGTCT \\
\hline BI184876 & Cytochrome P450 aromatase (CYP19) & AGTGGCTGAGGCATAAATTGAGAGA & TCCACAAAACGTGCTTTGAGAAAAA \\
\hline BI184877 & Glutathione S-transferase (GSTP) & AAGCAACCGTTATCTTCCTGCATTT & AATAGTTTCTTGGTCCCACAACACC \\
\hline BI185002 & GTP-binding protein RAB22A & AGAGCTCСTCTGCCTTCCСTTG & CACCAACTTCACTTCAGCGCTTTAT \\
\hline BI185003 & H. sapiens (TL27) mRNA from (PC3) cell line & GCCTGTTTCTTTITAGATCCCCGATA & ACAGGGACAAATATCACATCCCAAT \\
\hline $\mathrm{BI} 185028$ & $\mathrm{H} 1$ histone family, member $4(\mathrm{H} 1 \mathrm{~F} 4)$ & ССGCTGTACTTTTTGCTTTTCCTG & CTTTCTTAGCTTTGGGCTTGGCTTC \\
\hline BI185061 & Homo sapiens cDNA FLJ23553 & AAAAGAATCAAGAAGTGAAGCTGGG & TGGAGGTACTTTATTGGGGATAGGA \\
\hline BI185105 & MHC class I antigen PD1 (SLA1) & AGGCTGCAGGCAGTGACAGT & TGCTGCTGCAGAAACAGGAG \\
\hline BI185137 & Serine carboxypeptidase 1 precursor (HSCP1) & GGTGTTCAGGACATAGGGATGAGTG & ССTCTGTGGCAACTTGGAAATCAT \\
\hline BI185217 & Sus scrofa CC chemokine receptor genes & ССССAGTCTGTTTTTATTTGGCAGT & TTGGGGCATGTCATTGGTAATGTAT \\
\hline BI185259 & No match & ATGGAGTCCCAGAAAGCGTAACTGT & AGCAAGGATTTGATCCTGCCTAGAG \\
\hline BI185343 & ATP synthase gamma-subunit (L-type) & ACAGCCTGGCCAACATCATC & GGCACCGGAGATGATTTCAA \\
\hline BI185380 & Selenium binding protein 1 (SELENBP1) & TGAGGTTTTGACATTGTTAGCAGCA & СTCСATGTTCTGAGCCATCCTTTC \\
\hline BI185480 & Porcine endogenous retrovirus PERV & GCAAACAGCAAGAGGATTTTTATTTCA & GGCCTGTTTTACGTCCTTTCACCTA \\
\hline BI185578 & No match & СCTCATACCAAGTGCCGGAATTTAT & ATGATCATCAAAGTGGACCCTTGAA \\
\hline BI185839 & Fibronectin (FN precursor) & TATTGCGGGCCAGACAGTTAAGTAG & TCCCGAGAGTAAAATTTCCAAGCTG \\
\hline BI185976 & Sus scrofa CC chemokine receptor genes & CACAGCTGTAGCAACACCAGATCTTT & CCTGGTGAACTCAGGAGGGAATATG \\
\hline BI186028 & Peroxiredoxin 3 (PRDX3) & ACATTGAGCACGTCAAGAAAGAAGA & СTTCTGATCAGGGGTCCTGAAGATT \\
\hline BI186221 & Protein p8 (candidate of metastasis 1) & AGCCTCAGTCACGTGGGGTA & GAGACAGGACTGCGGAGGAA \\
\hline BI186223 & Human chromosome 1q21 BAC 236c22 & САСССТСССТСССGTTATTC & СТСТССTGCAAGGCACGTCT \\
\hline BI186431 & Calpain I light subunit & CTTGGCCTGAATGCACGAA & GTGTCAGGGGTGGCATCTTC \\
\hline M36513 & Follistatin & GTCTGTGGGCTGGATGGGAAA & ATCAAGGAAATCATGAATGGAAGCA \\
\hline U53020 & Steroidogenic acute regulatory protein (STAR) & CAGACCCAGGTGGACTTTGC & CGACACACACCACTGCAACA \\
\hline
\end{tabular}

for RH and/or SCHP mapping, primarily because of amplification of PCR fragments of similar size from pig and mouse/ hamster, and secondarily because of the presence of large introns in the swine genomic sequences.
The UCSC Human Genome Browser (http://genome.ucsc. edu/) represents a useful tool for predicting the physical position of human orthologues to swine expressed sequences. Our empirical mapping results agreed in $85 \%$ of the cases 
Table 2 PCR conditions for generating pig-specific amplicons, mapping results and comparative mapping with the human genome.

\begin{tabular}{|c|c|c|c|c|c|c|c|c|c|c|c|}
\hline \multirow[b]{2}{*}{$\begin{array}{l}\text { GenBank } \\
\text { accession } \\
\text { no. }{ }^{1}\end{array}$} & \multirow[b]{2}{*}{$\begin{array}{l}\text { PCR } \\
\text { programme }^{2}\end{array}$} & \multirow[b]{2}{*}{$\begin{array}{l}\text { Fragment } \\
\text { size }(b p)^{3}\end{array}$} & \multicolumn{2}{|l|}{$\mathrm{SCHP}^{4}$} & \multicolumn{3}{|l|}{$\mathrm{RH}^{5}$} & \multirow{2}{*}{$\begin{array}{l}\text { Reported } \\
\text { linkage } \\
\text { group } \\
\text { position } \\
(\mathrm{SSC})(\mathrm{cM})^{6}\end{array}$} & \multirow{2}{*}{$\begin{array}{l}\text { Reported } \\
\text { cytogenic } \\
\text { position } \\
\left(\mathrm{SSC}^{7}\right.\end{array}$} & \multirow{2}{*}{$\begin{array}{l}\text { Position of } \\
\text { human } \\
\text { orthologue } \\
(\mathrm{HSA})^{8}\end{array}$} & \multirow{2}{*}{$\begin{array}{l}\text { Expected } \\
\text { cytogenic } \\
\text { position } \\
(\mathrm{HSA})^{9}\end{array}$} \\
\hline & & & $\begin{array}{l}\text { Map } \\
\text { location }\end{array}$ & $\mathrm{R}$ & $\begin{array}{l}\text { Closest } \\
\text { marker }\end{array}$ & LOD & SSC & & & & \\
\hline BI181686 & OPT65 & 382 & - & - & SW1417 & 9.25 & 1 & (1) 44.1 & $1 \mathrm{p} 2.2-2.3$ & $6 q 23.2$ & $6 q$ \\
\hline Bl184876 & OPT65 & 378 & - & - & SWR702 & 8.92 & 1 & (1) 67.6 & $1 q 1.6-1.7$ & $15 q 21.2$ & $15 q 22$ \\
\hline BI183514 & OPT65 & 210 & - & - & SW2551 & 13.16 & 1 & (1) 95.8 & $1 \mathrm{q} 2.8$ & - & $9 q 22-31$ \\
\hline BI182064 & OPT65 & 410 & - & - & SW1311 & 7.72 & 1 & (1) 100.8 & $1 q 2.9-2.11$ & $9 q 31.3$ & $9 q 22-31$ \\
\hline AW584057 & OPT65 & 416 & - & - & SWR783 & 6.52 & 2 & (2) 23.7 & $2 p 1.6-1.7$ & $11 q 12.3$ & 11 \\
\hline Bl181791 & OPT62 & 192 & $5 p 11-15$ & 1.00 & SW747 & 3.62 & 2 & (2) 60.6 & $2 q 2.1$ & $22 q 13.33$ & $5 q / 19 p(22 q)$ \\
\hline AW584050 & OPT60 & 199 & - & - & 50010 & 14.83 & 2 & (2) 77.9 & $2 q 2.1-2.2$ & $2 q 33.1$ & $5 q / 19 p$ \\
\hline Bl182474 & OPT65 & 376 & - & - & SW1695 & 13.16 & 2 & (2) 80.6 & $2 q 2.1-2.8$ & $5 q 22.3$ & $5 q / 19 p$ \\
\hline BI181480 & OPT65 & 381 & - & - & S0335 & 6.40 & 3 & (3) 12.4 & $3 p 1.7$ & $7 q 22.1$ & $7 p 15-q 22$ \\
\hline BI185976 & OPT65 & 307 & - & - & SW833 & 6.63 & 3 & (3) 17.3 & $3 p 1.7$ & - & $16 p$ \\
\hline B|186221 & OPT65 & 100 & - & - & SW1443 & 6.25 & 3 & (3) 40.1 & $3 p 1.4$ & $16 p 12.1$ & $16 p$ \\
\hline BI183694 & OPT65 & 401 & $3 q 21-27$ & 0.83 & $I L 1 B$ & 4.30 & 3 & (3) 53.7 & $3 q 1.1-2.4$ & $2 \mathrm{p} 22.2$ & $2 p-q 21$ \\
\hline Bl182751 & OPT65 & 399 & - & - & 50002 & 8.75 & 3 & (3) 102.2 & $3 q 2.5-2.6$ & $2 \mathrm{p} 24.1$ & $2 p 23-24$ \\
\hline BI181040 & OPT65 & 387 & - & - & SWR362 & 10.32 & 4 & (4) 55.9 & $4 q 1.1-1.2$ & $8 q 22.1$ & $8 q$ \\
\hline BI186223 & OPT65 & 102 & - & - & SWR362 & 8.44 & 4 & (4) 55.9 & $4 q 1.1-1.2$ & $8 q 22.1$ & $8 q$ \\
\hline Bl184392 & OPT65 & 137 & - & - & SWR362 & 9.45 & 4 & (4) 55.9 & $4 q 1.1-1.2$ & $8 q 22.1$ & $8 q$ \\
\hline Bl184862 & OРT65 & 182 & - & - & SWR362 & 8.76 & 4 & (4) 55.9 & $4 q 1.1-1.2$ & $8 q 22.1$ & $8 q$ \\
\hline BI184415 & OPT62 & 112 & - & - & SW589 & 19.48 & 4 & (4) 74.4 & $4 q 1.6-2.1$ & - & $1 p 21-q 25$ \\
\hline AW574381 & OPT65 & 401 & $\begin{array}{l}\text { 1p21/ } \\
\text { q23-27 }\end{array}$ & 0.74 & SW1996 & 3.71 & 4 & (4) 77.0 & $4 q 1.6-2.1$ & $5 p 15.31$ & $\begin{array}{l}1 p 21-q 25 \\
(7 q / 9 / 14 q / 18)\end{array}$ \\
\hline BI181228 & OPT65 & 403 & - & - & SW512 & 14.49 & 4 & (4) 80.5 & $4 q 1.6-2.1$ & $1 q 21.3$ & $1 p 21-q 25$ \\
\hline BI185380 & OPT65 & 394 & - & - & SW512 & 15.10 & 4 & (4) 80.5 & $4 q 1.6-2.1$ & $1 q 21.3$ & $1 p 21-q 25$ \\
\hline Bl181384 & OPT65 & 330 & - & - & SW512 & 10.26 & 4 & (4) 80.5 & $4 q 1.6-2.1$ & - & $1 p 21-q 25$ \\
\hline BI181041 & OPT65 & 181 & - & - & $D K$ & 6.15 & 5 & (5) 72.3 & $5 p 1.1-1.2$ & $12 q 13.3$ & $12 / 22 q$ \\
\hline BI182045 & OPT65 & 370 & $5 p 11-15$ & 1.00 & $D K$ & 4.64 & 5 & (5) 72.3 & $5 p 1.1-1.2$ & $22 q 12.3$ & $12 / 22 q$ \\
\hline BI183974 & TD1 & 389 & - & - & $D K$ & 6.51 & 5 & (5) 72.3 & $5 p 1.1-1.2$ & $22 q 12.3$ & $12 / 22 q$ \\
\hline Bl182727 & OPT62 & 161 & - & - & SW1134 & 9.87 & 5 & (5) 80.8 & $5 p 1.1-1.2$ & - & $12 / 22 q$ \\
\hline BI181067 & OPT60 & 101 & - & - & SW963 & 9.45 & 5 & (5) 95.5 & $5 q 1.2$ & $12 p 13.31$ & 12 \\
\hline Bl181785 & OPT65 & 207 & - & - & SW1383 & 12.11 & 5 & (5) 125.0 & $5 q 2.3$ & $12 q 22$ & 12 \\
\hline BI181891 & OPT65 & 378 & - & - & SW1057 & 13.76 & 6 & (6) 47.1 & $6 p 1.4-1.5$ & $16 q 13$ & $16 q$ \\
\hline BI184629 & OPT65 & 306 & - & - & SW1355 & 6.01 & 6 & (6) 83.3 & $6 q 2.2-2.3$ & $1 \mathrm{p} 36.23$ & $1 p 22-36$ \\
\hline BI184356 & OPT65 & 397 & - & - & SW709 & 17.39 & 6 & (6) 89.3 & $6 \mathrm{q} 2.5-2.6$ & 1p36.12 & $1 \mathrm{p} 22-36$ \\
\hline BI183769 & OPT65 & 416 & - & - & SW322 & 21.13 & 6 & (6) 149.8 & $6 \mathrm{q} 3.1-3.5$ & $1 \mathrm{p} 32.3$ & $1 \mathrm{p} 22-36$ \\
\hline BI185578 & OPT65 & 398 & $6 q 32-35$ & 0.75 & SW1069 & 3.26 & 6 & (6) 155.2 & $6 \mathrm{q} 3.5$ & - & $1 \mathrm{p} 22-36$ \\
\hline BI184678 & OPT65 & 388 & - & - & SW2466 & 11.25 & 6 & (6) 164.6 & $6 \mathrm{q} 3.5$ & - & 1p22-36 \\
\hline BI183427 & OPT65 & 406 & - & - & SW2155 & 16.04 & 7 & (7) 32.9 & $7 p 1.1-1.2$ & $6 p 22.1$ & $14 q / 15 q$ \\
\hline BI181293 & OPT65 & 393 & $7 p 11 / 13$ & 0.84 & SSC11FO2 & 6.33 & 7 & (7) 57.7 & $7 p 1.1-q 1.1$ & - & $6 p / 15 q$ \\
\hline $\mathrm{BI} 185028$ & OPT65 & 406 & - & - & SSC11FO2 & 14.55 & 7 & (7) 57.7 & $7 p 1.1-q 1.1$ & $6 p 22.2$ & $6 p / 15 q$ \\
\hline BI185105 & OPT62 & 156 & - & - & SSC25E05 & 7.68 & 7 & (7) 57.7 & $7 p 1.1-q 1.1$ & $6 p 22.1$ & $6 p / 15 q$ \\
\hline BI181249 & OPT65 & 414 & $7 q 12-23 / q 26$ & 0.92 & SW859 & 5.07 & 7 & (7) 75.3 & $7 q 1.3-1.4$ & - & $6 p / 15 q$ \\
\hline BI184877 & OРT65 & 399 & - & - & SW859 & 6.00 & 7 & (7) 75.3 & $7 q 1.3-1.4$ & $6 p 12.2$ & $6 p / 15 q$ \\
\hline AW507066 & OPT65 & 410 & - & - & SWR1210 & 20.67 & 7 & (7) 82.8 & $7 q 1.3-1.4$ & $15 q 23.1$ & $6 p / 15 q$ \\
\hline BI181803 & OPT65 & 401 & - & - & SWR1210 & 14.68 & 7 & (7) 82.8 & $7 q 1.3-1.4$ & $15 q 24.1$ & $6 p / 15 q$ \\
\hline BI182567 & OPT65 & 396 & - & - & SW255 & 12.96 & 7 & (7) 85.6 & $7 q 2.1$ & $14 q 11.2$ & $14 q / 15 q$ \\
\hline BI181194 & OPT65 & 371 & $1 q 23-27$ & 0.78 & SW2537 & 3.03 & 7 & (7) 139.5 & $7 q 2.5-2.6$ & $3 q 27.2$ & $\begin{array}{l}14 q / 15 q \\
(7 q / 9 / 14 q / 18)\end{array}$ \\
\hline BI183240 & OPT65 & 377 & $7 q 12-23 / 26$ & 0.91 & SSC12BO9 & 3.37 & 7 & (7) 156 & $7 q 2.5-2.6$ & $14 q 32.33$ & $14 q / 15 q$ \\
\hline
\end{tabular}


Table 2 Continued.

\begin{tabular}{|c|c|c|c|c|c|c|c|c|c|c|c|}
\hline \multirow{2}{*}{$\begin{array}{l}\text { GenBank } \\
\text { accession } \\
\text { no. }{ }^{1}\end{array}$} & \multirow[b]{2}{*}{$\begin{array}{l}\text { PCR } \\
\text { programme } 2\end{array}$} & \multirow[b]{2}{*}{$\begin{array}{l}\text { Fragment } \\
\text { size }(b p)^{3}\end{array}$} & \multicolumn{2}{|l|}{$\mathrm{SCHP}^{4}$} & \multicolumn{3}{|l|}{$\mathrm{RH}^{5}$} & \multirow{2}{*}{$\begin{array}{l}\text { Reported } \\
\text { linkage } \\
\text { group } \\
\text { position } \\
(\mathrm{SSC})(\mathrm{cM})^{6}\end{array}$} & \multirow{2}{*}{$\begin{array}{l}\text { Reported } \\
\text { cytogenic } \\
\text { position } \\
(\mathrm{SSC})^{7}\end{array}$} & \multirow{2}{*}{$\begin{array}{l}\text { Position of } \\
\text { human } \\
\text { orthologue } \\
(\mathrm{HSA})^{8}\end{array}$} & \multirow{2}{*}{$\begin{array}{l}\text { Expected } \\
\text { cytogenic } \\
\text { position } \\
(\mathrm{HSA})^{9}\end{array}$} \\
\hline & & & $\begin{array}{l}\text { Map } \\
\text { location }\end{array}$ & $\mathrm{R}$ & $\begin{array}{l}\text { Closest } \\
\text { marker }\end{array}$ & LOD & SSC & & & & \\
\hline BI183574 & OPT65 & 434 & - & - & SW2521 & 8.13 & 8 & (8) 23.1 & $8 p 2.3$ & - & $4 p-q 31$ \\
\hline AW507072 & OPT65 & 419 & $8 p 12-21$ & 0.78 & SW206 & 4.00 & 8 & (8) 55.4 & $8 \mathrm{p} 2.1$ & $17 p 11.2$ & $4 p-q 31$ \\
\hline BI181579 & OPT60 & 133 & $8 p 21-23$ & 0.92 & SW206 & 5.85 & 8 & (8) 55.4 & $8 \mathrm{p} 2.1$ & $4 p 14$ & $4 p-q 31$ \\
\hline BI182250 & TD1 & 135 & $1 q 11-17$ & 0.92 & SW1924 & 3.91 & 8 & (8) 59.3 & $8 p 1.1-1.2$ & $4 q 31.3$ & $\begin{array}{l}4 p-q 31 \\
(15 q / 18)\end{array}$ \\
\hline BI183145 & OPT65 & 396 & - & - & SW1671 & 13.13 & 8 & (8) 99.0 & $8 q 2.5$ & $4 q 23$ & $4 p-q 31$ \\
\hline BI184390 & OPT65 & 401 & - & - & SWR68 & 6.86 & 9 & (9) 4.0 & $9 \mathrm{p} 2.4$ & $11 \mathrm{p} 15.4$ & 11 \\
\hline BI182164 & OPT65 & 378 & - & - & SW511 & 13.38 & 9 & (9) 70.4 & $9 \mathrm{p} 1.1-1.2$ & $11 q 23.3$ & 11 \\
\hline BI184102 & OPT65 & 375 & - & - & SW1615 & 8.39 & 9 & (9) 72.6 & $9 \mathrm{p} 1.1-1.2$ & $11 \mathrm{q} 24.1$ & 11 \\
\hline BI183910 & OPT65 & 396 & - & - & S0119 & 9.40 & 9 & (9) 83.3 & $9 q 1.1-1.2$ & $1 q 32.1$ & $1 q 31-41 / 11$ \\
\hline BI181696 & OPT65 & 397 & - & - & SW830 & 10.93 & 10 & (10) 0.0 & $10 p 1.6$ & $1 q 31.2$ & $1 q$ \\
\hline BI181240 & OPT65 & 401 & - & - & SSC25AO2 & 6.79 & 10 & (10) 67.5 & $10 \mathrm{q} 1.3$ & $9 p 21.1$ & $9 / 10$ \\
\hline BI181277 & TD1 & 113 & $10 q 11-12$ & 0.75 & SSC10G07 & 5.11 & 10 & (10) 67.5 & $10 q 1.3$ & $10 q 23.31$ & $9 / 10$ \\
\hline BI182214 & OPT65 & 388 & - & - & SSC10G07 & 6.39 & 10 & (10) 67.5 & $10 \mathrm{q} 1.3$ & $10 q 23.31$ & $9 / 10$ \\
\hline BI182422 & OPT65 & 391 & - & - & SW1829 & 8.33 & 10 & (10) 90.2 & $10 q 1.3-1.4$ & $10 p 12.2$ & $9 / 10$ \\
\hline BI181366 & OPT65 & 399 & $10 q 14-16$ & 1.00 & SW305 & 5.99 & 10 & (10) 94.5 & $10 q 1.3-1.4$ & - & $9 / 10$ \\
\hline BI185217 & OPT65 & 408 & $10 q 17$ & 1.00 & SW305 & 4.35 & 10 & (10) 94.5 & $10 q 1.3-1.4$ & - & $9 / 10$ \\
\hline BI184598 & TD1 & 133 & $10 q 14-16$ & 0.72 & SWR67 & 4.82 & 10 & (10) 122.0 & $10 q 1.7$ & 10p15.1 & $10 p$ \\
\hline BI185343 & OPT62 & 185 & - & - & SWR67 & 6.24 & 10 & (10) 122.0 & $10 \mathrm{q} 1.7$ & $14 q 22.2$ & $9 / 10$ \\
\hline BI183661 & OPT62 & 112 & - & - & SSC6EO9 & 9.35 & 11 & (11) 16.3 & $11 p 1.3$ & $13 q 14.11$ & $13 q$ \\
\hline BI185480 & OPT65 & 394 & $12 \mathrm{p} 11-13$ & 0.92 & SW943 & 13.76 & 12 & (12) 62.8 & $12 \mathrm{p} 1.1-1.4$ & - & 17 \\
\hline BI185061 & OPT65 & 140 & $12 p 11-13$ & 0.92 & SW874 & 15.81 & 12 & (12) 64.7 & 12p1.1-1.4 & $17 q 21.32$ & 17 \\
\hline BI180977 & OPT65 & 441 & - & - & SWR390 & 13.81 & 12 & (12) 70.5 & $12 p 1.1$ & $17 q 21.33$ & 17 \\
\hline BI181144 & OPT65 & 378 & - & - & SWR390 & 21.72 & 12 & (12) 70.5 & $12 p 1.1$ & $17 q 21.33$ & 17 \\
\hline BI181761 & OPT65 & 380 & - & - & SWR390 & 12.22 & 12 & (12) 70.5 & $12 p 1.1$ & $17 q 21.33$ & 17 \\
\hline BI181387 & OPT65 & 412 & $5 p 11-15$ & 0.93 & 50090 & 2.23 & 12 & (12) 80.2 & $12 \mathrm{p} 1.1-\mathrm{q} 1.3$ & $22 q 12.3$ & $17(12 / 22 q)$ \\
\hline BI184141 & OPT65 & 392 & $12 \mathrm{p} 11-13$ & 0.92 & SWC23 & 15.93 & 12 & (12) 95.8 & $12 p 1.1-q 1.3$ & $17 q 11.2$ & 17 \\
\hline BI181842 & OPT65 & 395 & - & - & 50288 & 7.43 & 13 & (13) 35.4 & $13 q 2.1$ & $20 q 13.12$ & 3 \\
\hline BI182004 & OPT65 & 411 & $13 q 23-41$ & 1.00 & SWR926 & 4.28 & 13 & (13) 56.4 & $13 q 2.3-2.4$ & $3 q 25.32$ & 3 \\
\hline Bl185003 & OPT65 & 401 & $13 q 23-41$ & 1.00 & $C P$ & 13.21 & 13 & (13) $90.9^{*}$ & $13 q 4.6-4.8$ & $3 q 25.1$ & 3 \\
\hline BI185137 & OPT65 & 351 & - & - & $C P$ & 16.79 & 13 & (13) $90.9^{*}$ & $13 q 4.6-4.8$ & $17 q 23.2$ & 3 \\
\hline BI181431 & OPT65 & 409 & - & - & SW245 & 16.97 & 14 & (14) 32.0 & $14 q 2.1$ & $12 q 24.31$ & $12 q / 22 q$ \\
\hline BI182401 & OPT62 & 157 & - & - & SW1109 & 11.14 & 14 & (14) 53.2 & $14 q 2.6-2.7$ & - & $1 p 22 / 10 q$ \\
\hline BI181263 & OPT65 & 143 & - & - & SW1333 & 11.68 & 14 & (14) 62.4 & $14 q 2.6-2.7$ & $10 q 24.32$ & $1 q 22 / 10 q$ \\
\hline BI182336 & OPT65 & 393 & - & - & SW2057 & 11.61 & 14 & (14) 62.4 & $14 q 2.6-2.7$ & $10 \mathrm{q} 24.32$ & $1 p 22 / 10 q$ \\
\hline BI186028 & OPT65 & 374 & - & - & SW55 & 13.89 & 14 & (14) 79.0 & $14 q 2.6-2.9$ & $10 q 26.11$ & $1 p 22 / 10 q$ \\
\hline BI182047 & OPT65 & 168 & - & - & $S W C 27$ & 8.22 & 14 & (14) 111.5 & $14 \mathrm{q} 2.9$ & $10 q 26.3$ & $1 \mathrm{p} 22 / 10 \mathrm{q}$ \\
\hline BI180991 & OPT65 & 414 & - & - & SW1562 & 8.50 & 15 & (15) 29.5 & $15 q 1.1-1.5$ & $2 q 14.1$ & $2 q / 4 q$ \\
\hline U53020 & OPT62 & 200 & - & - & SW1989 & 6.61 & 15 & (15) 57.9 & $15 q 1.5$ & $8 p 12$ & $2 q / 4 q$ \\
\hline BI182881 & OPT62 & 102 & - & - & 50088 & 6.32 & 15 & (15) 65.1 & $15 q 2.2$ & $2 q 24.3$ & $2 q / 4 q$ \\
\hline BI182556 & OPT65 & 379 & - & - & SW1263 & 9.29 & 15 & (15) 67.5 & $15 q 2.2$ & $4 q 22.1$ & $2 q / 4 q$ \\
\hline BI182232 & OPT65 & 390 & - & - & SW1316 & 6.03 & 15 & (15) 73.1 & $15 q 2.2$ & $2 q 33.1$ & $2 q / 4 q$ \\
\hline BI181421 & OPT65 & 239 & $4 q 21-23$ & 0.92 & SWR1002 & 3.56 & 15 & (15) 76.0 & $15 q 2.2$ & 1p12 & $2 q / 4 q(1 / 8 q)$ \\
\hline BI185839 & OPT65 & 409 & - & - & SW1683 & 15.41 & 15 & (15) 79.3 & $15 q 2.3-2.4$ & $2 q 35$ & $2 q / 4 q$ \\
\hline AW574403 & TD1 & 171 & - & - & SW2608 & 5.56 & 15 & (15) 95.0 & $15 q 2.5$ & $5 p 15.31$ & $2 q / 4 q$ \\
\hline BI182428 & OPT65 & 392 & - & - & SW742 & 9.92 & 16 & (16) 9.3 & $16 q 1.1-1.2$ & $5 p 15.1$ & 5 \\
\hline M36513 & OPT60 & 340 & $16 q 14 / 22-23$ & 0.92 & 50077 & 4.24 & 16 & (16) 33.2 & $16 q 1.4$ & $5 q 11.2$ & 5 \\
\hline
\end{tabular}


Table 2 Continued.

\begin{tabular}{|c|c|c|c|c|c|c|c|c|c|c|c|}
\hline \multirow{2}{*}{$\begin{array}{l}\text { GenBank } \\
\text { accession } \\
\text { no. }^{1}\end{array}$} & \multirow[b]{2}{*}{$\begin{array}{l}\text { PCR } \\
\text { programme } 2\end{array}$} & \multirow[b]{2}{*}{$\begin{array}{l}\text { Fragment } \\
\text { size }(b p)^{3}\end{array}$} & \multicolumn{2}{|l|}{$\mathrm{SCHP}^{4}$} & \multicolumn{3}{|l|}{$\mathrm{RH}^{5}$} & \multirow{2}{*}{$\begin{array}{l}\text { Reported } \\
\text { linkage } \\
\text { group } \\
\text { position } \\
(\mathrm{SSC})(\mathrm{CM})^{6}\end{array}$} & \multirow{2}{*}{$\begin{array}{l}\text { Reported } \\
\text { cytogenic } \\
\text { position } \\
(\mathrm{SSC})^{7}\end{array}$} & \multirow{2}{*}{$\begin{array}{l}\text { Position of } \\
\text { human } \\
\text { orthologue } \\
(\mathrm{HSA})^{8}\end{array}$} & \multirow{2}{*}{$\begin{array}{l}\text { Expected } \\
\text { cytogenic } \\
\text { position } \\
(\mathrm{HSA})^{9}\end{array}$} \\
\hline & & & $\begin{array}{l}\text { Map } \\
\text { location }\end{array}$ & $\mathrm{R}$ & $\begin{array}{l}\text { Closest } \\
\text { marker }\end{array}$ & LOD & SSC & & & & \\
\hline BI181787 & OPT65 & 385 & - & - & SW1897 & 7.18 & 16 & (16) 86.2 & $16 \mathrm{q} 2.3$ & $5 q 33.1$ & 5 \\
\hline BI182872 & OPT65 & 425 & - & - & SW1897 & 6.66 & 16 & (16) 86.2 & $16 \mathrm{q} 2.3$ & $5 q 33.1$ & 5 \\
\hline BI186431 & OPT65 & 103 & - & - & SW1897 & 8.70 & 16 & (16) 86.2 & $16 \mathrm{q} 2.3$ & $5 q 33.1$ & 5 \\
\hline BI182007 & OPT65 & 421 & - & - & SW24 & 14.73 & 17 & (17) 23.3 & $17 q 1.1-1.2$ & - & 20 \\
\hline BI184717 & OPT65 & 405 & $17 q 21-23$ & 1.00 & SW1031 & 4.46 & 17 & (17) 63.4 & $17 q 2.1$ & $20 q 13.12$ & 20 \\
\hline BI185002 & OPT65 & 386 & - & - & S0332 & 13.99 & 17 & (17) 88.7 & $17 q 2.3$ & $20 q 13.32$ & 20 \\
\hline BI181663 & OPT62 & 142 & $17 q 21-23$ & 0.80 & SW2431 & 5.08 & 17 & (17) 94.0 & $17 q 2.3$ & $20 q 13.12$ & 20 \\
\hline BI185259 & OPT65 & 401 & - & - & S0177 & 15.16 & 18 & (18) 55.3 & $17 q 2.1$ & - & 7 \\
\hline BI182487 & OPT65 & 424 & $\mathrm{Xq11-12}$ & 0.86 & SW1549 & 2.22 & $x$ & (X) 11.9 & Xp2.4 & - & $x$ \\
\hline BI184594 & ОРТ65 & 402 & - & - & SSC13B11 & 7.18 & $x$ & (X) 74.4 & Xp2.1-2.2 & Xp11.3 & $x$ \\
\hline
\end{tabular}

PCR, polymerase chain reaction; SCHP, somatic cell hybrid panel; $\mathrm{RH}$, radiation hybrid

${ }^{1}$ Accession no. for the EST sequence used for primer design.

${ }^{2} \mathrm{PCR}$ programmes used for amplification were as follows: OPT 60,40 cycles of $95^{\circ} \mathrm{C} / 30 \mathrm{~s}, 60{ }^{\circ} \mathrm{C} / 45 \mathrm{~s}$ and $72{ }^{\circ} \mathrm{C} / 90 \mathrm{~s} ;$ OPT62, $40 \mathrm{cycles}$ of $95{ }^{\circ} \mathrm{C} /$ $30 \mathrm{~s}, 62{ }^{\circ} \mathrm{C} / 45 \mathrm{~s}$ and $72{ }^{\circ} \mathrm{C} / 90 \mathrm{~s}$; OPT 65,40 cycles of $95{ }^{\circ} \mathrm{C} / 30 \mathrm{~s}, 65^{\circ} \mathrm{C} / 45 \mathrm{~s}$ and $72{ }^{\circ} \mathrm{C} / 90 \mathrm{~s}$; TD1, 10 cycles of $94{ }^{\circ} \mathrm{C} / 60 \mathrm{~s}, 64{ }^{\circ} \mathrm{C}\left(-1{ }^{\circ} \mathrm{C} / \mathrm{cycle}\right) /$ $60 \mathrm{~s}, 72{ }^{\circ} \mathrm{C} / 60 \mathrm{~s}$, plus 30 cycles of $93{ }^{\circ} \mathrm{C} / 30 \mathrm{~s}, 55^{\circ} \mathrm{C} / 45 \mathrm{~s}, 72{ }^{\circ} \mathrm{C} / 45 \mathrm{~s}$.

${ }^{3}$ Base-pair size of the porcine PCR fragment amplified with the corresponding primers listed in Table 1.

${ }^{4}$ Results obtained after submitting the data to the INRA database-R refers to the correlation coefficients between each marker and the chromosomal region(s) listed, estimated according to Chevalet \& Corpet (1986).

${ }^{5}$ Closest $\mathrm{RH}$-linked marker, the LOD score for the estimate and the porcine chromosome where the respective $\mathrm{RH}$ group is located.

${ }^{6}$ Linkage position of the closest RH-linked marker also present in the porcine linkage map Rohrer et al. (1996) or according to Marklund et al. (1996), where marked by $a^{* *}$.

${ }^{7}$ Location of the closest RH-linked marker also present in the porcine cytogenetic map.

${ }^{8}$ Position of the corresponding human ortholgous sequence according to the UCSC Genome Browser (http://genome.ucsc.edu/), assembly of July 2003.

${ }^{9}$ Expected cytogenic position (HSA) according to Goureau et al. (1996) (see http://www.toulouse.inra.fr/lgc/pig/compare/compare.htm) based on the RH and SCHP map-location (data in parentheses refers to the expected position based on the SCHP results that were discordant of the RHmapping results).

with the chromosomal locations predicted (Table 2). In the remaining cases, the observed disagreements may be the result of shuffling of small terminal regions of ancestral chromosomes, which cannot be detected by chromosome painting techniques, and result in disruptions of the many large synteny blocks conserved between human and pig chromosomes, as previously reported in other studies (Messer et al. 1997; Larsen et al. 1999). In addition, discrepancies may be caused by mapped loci belonging to gene families and/or being duplicated loci that were physically separated during the independent evolution of the human and porcine genomes. Further characterization of the amplicons used for mapping these respective ESTs will be necessary to clarify these issues.

Identifying the underlying genes and respective polymorphisms regulating complex traits such as ovulation rate in swine has been a major challenge (see Pomp et al. 2001; Rohrer 2004). Integrating phenotypic data with mapping and gene expression experiments provides a powerful approach to dissect the nature of mechanisms controlling complex traits
(Pomp et al. 2004). All of the genes mapped in this study were found to be putatively differentially expressed in the ovaries and ovarian follicles between a swine line selected for high ovulation rate and its control line (Caetano et al. 2004). Quantitative and/or qualitative changes in mRNA expression may be the result of cis-acting allelic variations at the specific gene (i.e. a QTL) or conversely, may result from trans-acting mutations at QTL that control the particular gene (Yvert et al. 2003; Pomp et al. 2004). Several porcine genomic regions have been found to contain QTL affecting ovulation rate, and twelve of the genes we mapped in this study are located in those regions (Table 3 ). These differentially expressed genes found to map to regions containing QTL that are associated with differences in ovulation rate in swine become immediate positional candidates. Of particular interest are the genes of yet unknown identity and/or function (i.e. BI182164). This approach is somewhat limited because of the very broad confidence intervals attributed to most QTL localizations. Concurrently, map positions of the ESTs evaluated in this study 
Table 3 Previously reported QTL affecting ovulation rate in swine and associated EST-derived markers.

\begin{tabular}{|c|c|c|c|c|c|}
\hline $\mathrm{SSC}^{1}$ & Position $(\mathrm{cM})^{2}$ & Reference & $\begin{array}{l}\text { GenBank } \\
\text { accession no. }\end{array}$ & $\begin{array}{l}\text { Closest } \\
\text { RH marker }{ }^{4}\end{array}$ & $\begin{array}{l}\text { Reported linkage } \\
\text { group position }(\mathrm{CM})^{5}\end{array}$ \\
\hline 3 & $36[3: 70]$ & Rohrer et al. (1999) & BI186221 & SW1443 & 40.1 \\
\hline \multirow[t]{2}{*}{4} & $80(32)$ & Rathje et al. (1997) & BI181384 & SW512 & 80.5 \\
\hline & & & BI185380 & & \\
\hline \multirow[t]{2}{*}{8} & $50(29)$ & Wilkie et al. (1996) & AW507072 & SW206 & 55.4 \\
\hline & & & BI181579 & & \\
\hline \multirow[t]{2}{*}{8} & $50(101)$ & Wilkie et al. (1999) & AW507072 & SW206 & 55.4 \\
\hline & & & BI181579 & & \\
\hline 8 & 105 & Rathje et al. (1997) & - & - & - \\
\hline 15 & & & BI182881 & & \\
\hline 15 & 79 [53:101] & Rohrer et al. (1999) & Bl185839 & SW1683 & 79.3 \\
\hline 15 & $100(107)$ & Wilkie et al. (1999) & AW574403 & SW2608 & 95.0 \\
\hline
\end{tabular}

${ }^{1}$ Porcine chromosome.

${ }^{2}$ Relative position in Kosambi centimorgans as reported based on maps developed by Rohrer et al. (1996). Numbers in parentheses are relative position as reported by the authors. Numbers in brackets indicate the mapping range of the detected QTL as reported by the authors.

${ }^{3}$ Genbank accession no. for the EST sequence used for primer design.

${ }^{4} \mathrm{RH}$-linked marker closest to the EST-derived marker.

${ }^{5}$ Linkage position of the closest RH-linked marker also present in the porcine linkage map Rohrer et al. (1996).

will likely become more refined as the number of loci in the RH database, against which two-point analyses can be conducted with the current set of ESTs, continues to grow.

Disagreements between mapping results using the RH and SCH panels were observed with six markers (Table 2). This was also observed in other experiments that involved mapping markers with these two methodologies (Lahbib-Mansais et al. 2000, 2003; Tuggle et al. 2003). The underlying cause of the discrepancy was most likely a low LOD score for the RH panel (RHP), and thus the SCHP should be used as the best predicted map position, especially if it matches predictions based on human data. Amplification of multiple fragments from paralogous gene families and/or duplicated loci has been reported in other SCHP mapping studies (Caetano et al. 1999) and may also be the cause of the observed discrepancies between the RH and SCH mapping results. Seven of the mapped genes are represented by more than one EST/ primer-set (i.e. BI181787, BI182872 and BI186431). This redundancy was used retrospectively to check the robustness of the results. In these cases, mapping results based on different primer sets were in complete agreement, except for TIMP3 (BI181387, BI183974 and BI182045) where one of the markers mapped to SSC12 with the RHP with a low LOD score (2.23). This EST mapped to SSC5 using SCHP, in agreement with results from the other EST representing this gene.

\section{Acknowledgements}

The authors are grateful to Sara Olberding and Jackie Potts for laboratory assistance in this project. This work was partially supported by USDA-NRICGP 99-35205-8370 and by the National Science Foundation under Grant no. 0091900 (Nebraska EPSCOR infrastructure improvement grant). This work is published as paper number 14728 of the Journal Series, Nebraska Agricultural Experiment Station.

\section{References}

Caetano A.R., Shiue Y.L., Lyons L.A., Laughlin T.F., O’Brien S.J., Bowling A.T., and Murray J.D. (1999) A comparative gene map of the horse (Equus caballus). Genome Research 9, 1239- 49.

Caetano A.R., Johnson R.K., and Pomp D. (2003) Generation and sequence characterization of a normalized cDNA library from swine ovarian follicles. Mammalian Genome 14, 65- 70.

Caetano A.R., Johnson R.K., Ford J.J., and Pomp D. (2004) Microarray profiling for differential gene expression in ovaries and ovarian follicles of pigs selected for increased ovulation rate. Genetics 168, 1529-37.

Cassady J.P., Johnson R.K., Pomp D., Rohrer G.A., Van Vleck L.D., Spiegel E.K., and Gilson K.M. (2001) Identification of quantitative trait loci affecting reproduction in pigs. Journal of Animal Science 79, 623-33. 
Chevalet C. and Corpet F. (1986) Statistical decision rules concerning synteny or independence between markers. Cytogenetics and Cell Genetics 43, 132- 9 .

Fahrenkrug S.C., Smith T.P., Freking B.A., et al. (2002) Porcine gene discovery by normalized cDNA-library sequencing and EST cluster assembly. Mammalian Genome 13, 475-8.

Fujii J., Otsu K., Zorzato F., de Leon S., Khanna V.K., Weiler J.E., O’Brien P.J., and MacLennan D.H. (1991) Identification of a mutation in porcine ryanodine receptor associated with malignant hyperthermia. Science $\mathbf{2 5 3}$, $448-51$.

Goureau A., Yerle M., Schmitz A., Riquet J., Milan D., Pinton P., Frelat G., and Gellin J. (1996) Human and porcine correspondence of chromosome segments using bi-directional chromosome painting. Genomics 36, $252-62$.

Grisart B., Farnir F., Karim L., et al. (2004) Genetic and functional confirmation of the causality of the DGAT1 K232A quantitative trait nucleotide in affecting milk yield and composition. Proceedings of the National Academy of Sciences of the United States of America 101, 2398- 403.

Johnson R.K., Nielsen M.K., and Casey D.S. (1999) Responses in ovulation rate, embryonal survival, and litter traits in swine to 14 generations of selection to increase litter size. Journal of Animal Science 77, 541- 57.

Lahbib-Mansais Y., Leroux S., Milan D., Yerle M., Robic A., Jiang Z., Andre C., and Gellin J. (2000) Comparative mapping between humans and pigs: localization of 58 anchorage markers (TOASTs) by use of porcine somatic cell and radiation hybrid panels. Mammalian Genome 11, 1098- 106.

Lahbib-Mansais Y., Tosser-Klopp G., Leroux S., Cabau C., Karsenty E., Milan D., Barillot E., Yerle M., Hatey F., and Gellin J. (2003) Contribution to high-resolution mapping in pigs with 101 type I markers and progress in comparative map between humans and pigs. Mammalian Genome 14, 275- 88.

Larsen N.J., Marklund S., Kelly K.A., Malek M., Tuggle C.K., Yerle M., and Rothschild M.F. (1999) New insights into porcine-human synteny conservation. Mammalian Genome 10, 488-91.

Marklund L., Johansson Moller M., Hoyheim B., Davies W., Fredholm M., Juneja R.K., Mariani P., Coppieters W., Ellegren H., and Andersson L. (1996) A comprehensive linkage map of the pig based on a wild pigLarge White intercross. Animal Genetics 27, 255- 69.

McPherron A.C. and Lee S.J. (1997) Double muscling in cattle due to mutations in the myostatin gene. Proceedings of the National Academy of Sciences of the United States of America 94, 12457- 61.

Messer L.A., Wang L., Tuggle C.K., et al. (1997) Mapping of the melatonin receptor 1a (MTNR1A) gene in pigs, sheep, and cattle. Mammalian Genome 8, 368-70.

Milan D., Jeon J.T., Looft C., et al. (2000) A mutation in PRKAG3 associated with excess glycogen content in pig skeletal muscle. Science $\mathbf{2 8 8}$, $1248-51$

Page B.T., Casas E., Heaton M.P., et al. (2002) Evaluation of single-nucleotide polymorphisms in CAPN1 for association with meat tenderness in cattle. Journal of Animal Science 80, 3077- 85.

Pomp D., Caetano A.R., Bertani G.R., Gladney C.D., and Johnson R.K. (2001) Applying functional genomics research to the study of pig reproduction. Reproduction Supplement 58, 277-92.

Pomp D., Allan M.F., and Wesolowski S.R. (2004) Quantitative genomics: exploring the genetic architecture of complex trait predisposition. Journal of Animal Science 82, 300- 12.
Quackenbush J., Liang F., Holt I., Pertea G., and Upton J. (2000) The TIGR Gene Indices: reconstruction and representation of expressed gene sequences. Nucleic Acids Research 28, 141-5.

Rathje T.A., Rohrer G.A., and Johnson R.K. (1997) Evidence for quantitative trait loci affecting ovulation rate in pigs. Journal of Animal Science 75, 1486- 94 .

Rink A., Santschi E.M., Eyer K.M., Roelofs B., Hess M., Godfrey M., Karajusuf E.K., Yerle M., Milan D., and Beattie C.W. (2002) A first-generation EST RH comparative map of the porcine and human genome. Mammalian Genome 13, 578- 87.

Robic A., Faraut T., Iannuccelli N., Lahbib-Mansais Y., Cantegrel V., Alexander L., and Milan D. (2003) A new contribution to the integration of human and porcine genome maps: 623 new points of homology. Cytogenetics and Genome Research 102, 100- 8.

Rohrer G.A. (2004) An overview of genomics research and its impact on livestock reproduction. Reproduction Fertility and Development 16, 47- 54.

Rohrer G.A., Alexander L.J., Hu Z., Smith T.P., Keele J.W., and Beattie C.W. (1996) A comprehensive map of the porcine genome. Genome Research 6, 371-91.

Rohrer G.A., Ford J.J., Wise T.H., Vallet J.L., and Christenson R.K. (1999) Identification of quantitative trait loci affecting female reproductive traits in a multigeneration Meishan-White composite swine population. Journal of Animal Science 77, 1385-91.

Rothschild M.F. (2003) From a sow's ear to a silk purse: real progress in porcine genomics. Cytogenetic and Genome Research 102, 95- 9.

Schadt E.E., Monks S.A., Drake T.A., et al. (2003) Genetics of gene expression surveyed in maize, mouse and man. Nature 422, 397- 402.

Tuggle C.K., Green J.A., Fitzsimmons C., et al. (2003) EST-based gene discovery in pig: virtual expression patterns and comparative mapping to human. Mammalian Genome 14, 565- 79.

Wayne M.L. and McIntyre L.M. (2002) Combining mapping and arraying: An approach to candidate gene identification. Proceedings of the National Academy of Sciences of the United States of America 99, 14903-6.

Wilkie P.J., Paszek A.A., Flickinger G.H., Rohrer G.A., Alexander L.J., Beattie C.W., and Schook L.B. (1996) Scan of eight porcine chromosomes for growth, carcass and reproductive traits reveals two likely quantitative trait loci. Animal Genetics 27 (Suppl. 2), 117-8 (Abstract).

Wilkie P.J., Paszek A.A., Beattie C.W., Alexander L.J., Wheeler M.B., and Schook L.B. (1999) A genomic scan of porcine reproductive traits reveals possible quantitative trait loci (QTLs) for number of corpora lutea. Mammalian Genome 10, 573- 8.

Yerle M., Echard G., Robic A., Mairal A., Dubut-Fontana C., Riquet J., Pinton P., Milan D., Lahbib-Mansais Y., and Gellin J. (1996) A somatic cell hybrid panel for pig regional gene mapping characterized by molecular cytogenetics. Cytogenetics and Cell Genetics 73, 194- 202.

Yerle M., Pinton P., Robic A., et al. (1998) Construction of a whole-genome radiation hybrid panel for high-resolution gene mapping in pigs. Cytogenetics and Cell Genetics 82, 182-8.

Yvert G., Brem R.B., Whittle J., Akey J.M., Foss E., Smith E.N., Mackelprang R., and Kruglyak L. (2003) Trans-acting regulatory variation in Saccharomyces cerevisiae and the role of transcription factors. Nature Genetics 35, 57-64. 